\title{
Implementação Computacional do Método de Floyd para Análise da Resposta Transitória em Reguladores
}

\author{
José Flávio Feiteira, José Luiz Guarino e Rodrigo Guerra de Souza* \\ Depto. VCE - Modelagem Computacional em Ciência e Tecnologia MCCT/EEIMVR/UFF. \\ 27255-125, Av. dos Trabalhadores 420 Vila Sta Cecília - Volta Redonda, RJ.
}

\section{RESUMO}

No presente trabalho é desenvolvida e aplicada uma metodologia, na qual calcula-se computacionalmente, e em ambiente Matlab, a resposta ao degrau unitário no domínio do tempo, dada inicialmente uma resposta no domínio da frequência (ganho e defasagem) de um regulador em malha fechada pelo método de Floyd para análise de sua resposta transitória. Conclui-se, a partir dos gráficos apresentados, que o método computacional gera resultados satisfatórios quando comparado com o modelo analítico.

Palavras-chave: Resposta Transitória, Reguladores, Método de Floyd.

INTRODUÇÃO: Na análise de resposta transitória dos reguladores, em geral, não há uma correlação direta entre a resposta do sistema no domínio da frequência e a resposta ao degrau unitário no domínio do tempo. O método de Floyd é conhecido por resolver este problema através de análise gráfica, entretanto o presente trabalho traz o desenvolvimento deste método implementado computacionalmente, ou seja, a partir de uma equação numérica, calcula-se a resposta ao degrau unitário no domínio do tempo $r(t)$ dada uma resposta no domínio da frequência $G(w)$ conhecidos o ganho e a defasagem de um sistema de segunda ordem sujeito à seguinte restrição:

$$
\lim _{w \rightarrow \infty} G(w)=0
$$

METODOLOGIA: Utilizando-se a transformada inversa de Fourier na resposta de frequência $G(w)$ do sistema, deduz-se a resposta ao degrau unitário no domínio do tempo $r(t)$ conforme a equação (2):

$$
r(t)=\frac{2}{\pi} \int_{0}^{\infty} G \cos \theta \frac{\sin (w t)}{w} d w
$$

onde, $G$ é o ganho, $\theta$ é a defasagem, e $w$ é a frequência em $\mathrm{rad} / \mathrm{s}$.

De posse da resposta no domínio da frequência do sistema de malha fechada, calcula-se a curva $G \cos \theta$, impondo-se a linearização por setores na banda de frequência $B$. O método de Floyd consiste na linearização desta curva, na qual $G \cos \theta$ é escrito na forma linear $B=a w+b$, onde $a$ e $b$ são, respectivamente, os coeficientes angular e linear a determinar. Após a linearização, obtem-se a equação numérica (3) para o cálculo da resposta ao degrau unitário no domínio do tempo $r(t)$, onde $S_{i}(w t)$ é a função Seno-integral.

$$
\begin{aligned}
r(t)=\frac{2}{\pi} \sum_{w=0}^{B}\left[\left(\frac{G_{j} \cos \left(\theta_{j}\right)-G_{i} \cos \left(\theta_{i}\right)}{w_{i}-w_{j}}\right)\left(\frac{\cos \left(w_{j} t\right)-\cos \left(w_{i} t\right)}{t}\right)\right]+ \\
+\left[\left(\frac{w_{i} G_{j} \cos \left(\theta_{j}\right)-w_{j} G_{i} \cos \left(\theta_{i}\right)}{w_{i}-w_{j}}\right)\left(S_{i}\left(w_{j} t\right)-S_{i}\left(w_{i} t\right)\right)\right]
\end{aligned}
$$

*Estudantes no MCCT/EEIMVR/UFF. E-mail: galloguarino@gmail.com e rodrigomct@ gmail.com 
IMPLEMENTAÇÃO: Dado um sistema de segunda ordem cuja equação analítica (4) no domínio da frequência é:

$$
G(s)=\frac{w_{n}^{2}}{s^{2}+2 \zeta w_{n} s+w_{n}^{2}}
$$

onde $\zeta=0.2$ e $w_{n}=1 \mathrm{rad} / \mathrm{s}$

Fazendo-se $s=j w$, temos os seguintes gráficos:

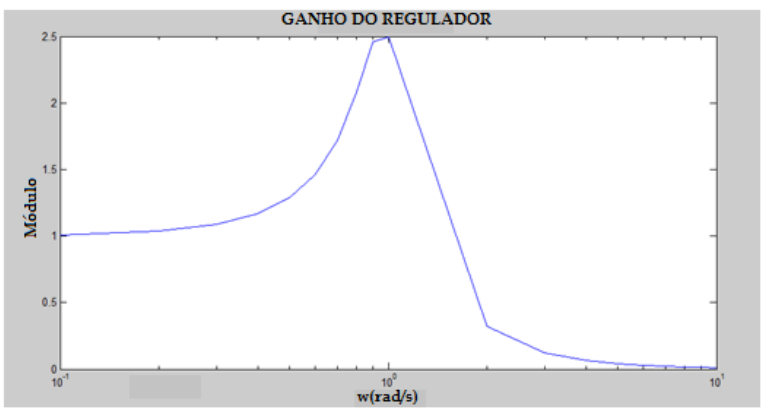

(a) Ganho

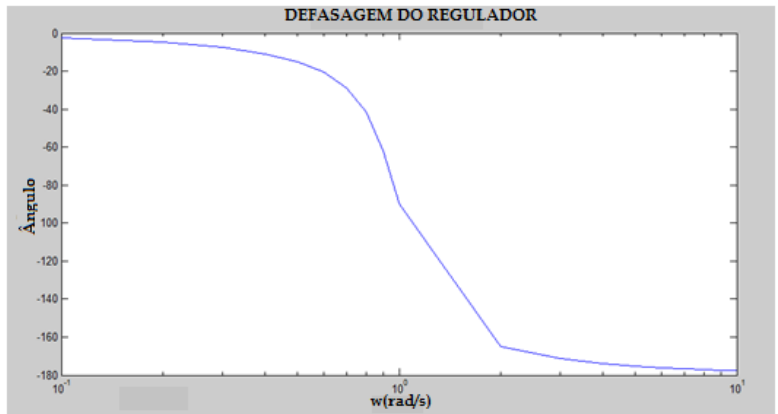

(b) Defasagem

Figura 1: Resposta de frequência num sistema de malha fechada

Caso o ganho $G$ seja dado em Decibéis, calcula-se $G^{\prime}=(10)^{\frac{G}{20}}$;

Caso a frequência $f$ seja dada em $H z$, calcula-se $w=2 \pi f$.

DISCUSSÃO E RESULTADOS: Com base nos resultados obtidos, ver figura (2), verifica-se que o Método de Floyd, implementado computacionalmente, apresenta resultados satisfatórios, porém com um pequeno retardo no tempo quando comparados com a solução analítica.

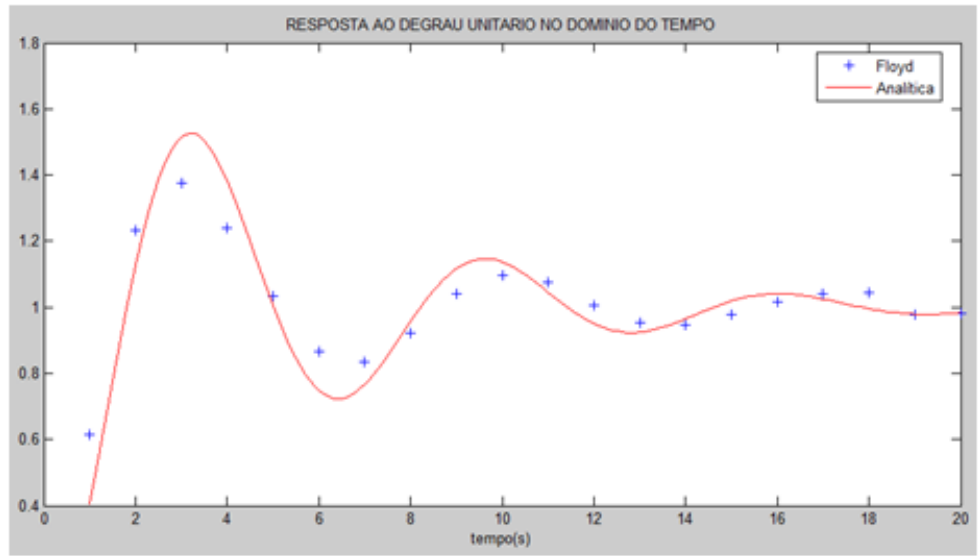

Figura 2: Resposta ao degrau unitário no domínio do tempo

Esse fenômeno ocorre, porque linearização da curva não considera a banda de frequência completa conforme os gráficos da figura (1)

\section{Referências}

[1] J.M.Gordon, Basic Automatic Control Theory, First Edition, June 1957, D.Van Nostrand Company, INC. Princeton, New Jersey. 